\title{
Muscle Contraction
}

\section{H. Lee Sweeney and David W. Hammers}

Department of Pharmacology and Therapeutics and the Myology Institute, University of Florida, College of Medicine, Gainesville, Florida 32610-0267

Correspondence: Isweeney@ufl.edu

\section{SUMMARY}

Muscle cells are designed to generate force and movement. There are three types of mammalian muscles_-skeletal, cardiac, and smooth. Skeletal muscles are attached to bones and move them relative to each other. Cardiac muscle comprises the heart, which pumps blood through the vasculature. Skeletal and cardiac muscles are known as striated muscles, because the filaments of actin and myosin that power their contraction are organized into repeating arrays, called sarcomeres, that have a striated microscopic appearance. Smooth muscle does not contain sarcomeres but uses the contraction of filaments of actin and myosin to constrict blood vessels and move the contents of hollow organs in the body. Here, we review the principal molecular organization of the three types of muscle and their contractile regulation through signaling mechanisms and discuss their major structural and functional similarities that hint at the possible evolutionary relationships between the cell types.

\section{Outline}

1 Introduction

2 The sarcomere of striated muscles

3 Thin filaments

4 Regulation of striated muscle contraction resides on the thin filament

5 Thick filaments

6 Myosin isoforms of muscle

7 Titin

8 Actin-myosin interactions within the sarcomere
9 Z- and M-lines function as cross-linking regions

10 Force transmission

11 Signaling roles of the sarcomere

12 Differences between cardiac and skeletal muscle

13 Differences between striated and smooth muscle

14 Conclusion

References 


\section{INTRODUCTION}

The evolution of contractile muscle provided higher organisms in the animal kingdom with the ability to be mobile within their environment. There are three types of muscle: skeletal, cardiac, and smooth. Myocytes are the cellular unit of muscle structure and contain high concentrations of specialized proteins that use chemical energy to generate mechanical force in the form of cellular contraction. Skeletal and cardiac muscle are referred to as striated muscle because of the visible organization of repeating units of contractile filaments, known as sarcomeres, into cylindrical bundles called myofibrils. In mature muscle fibers (striated muscle myocytes), most of the cellular volume is occupied by myofibril, leaving little room for nuclei and associated Golgi system, mitochondria, sarcoplasmic reticulum (SR; the specialized endoplasmic reticulum of striated muscle), glycogen granules, and other organelles/structures. In contrast, smooth muscles have large amounts of actin and myosin filaments that are not organized into sarcomeres. Unlike striated muscle cells, which are postmitotic, smooth muscle cells can proliferate under physiological and pathological conditions. Striated muscles are regulated by $\mathrm{Ca}^{2+}$, which is released from the SR and binds to troponin (Tn) on the actin filament. This event releases tropomyosin (Tm) from its position, which blocks the interaction of myosin heads with actin. However, smooth muscle does not contain $\mathrm{Tn}$, and instead contraction is regulated by the level of myosin regulatory light chain (RLC) phosphorylation.

\section{THE SARCOMERE OF STRIATED MUSCLES}

Depicted in Figure 1 is the sarcomere, which is the basic contractile unit of striated muscle. Sarcomeres are organized in series to make up a myofibril. The sarcomere is defined as spanning from Z-line to Z-line (described in detail below), only a few micrometers long, and consists of an A band containing myosin ("thick") filaments, which is flanked by two half I-bands made up of actin ("thin") filaments. This A band is the central region of the sarcomere, composed primarily of myosin filaments, the force-generating motor protein of skeletal, cardiac, and smooth muscle (reviewed by Sweeney and Holzbaur 2016). Muscle myosin, now referred to as myosin II or conventional myosin, was the first of many members discovered in the myosin superfamily of motor proteins (Odronitz and Kollmar 2007). An additional nonmuscle form of myosin II contributes to cytokinesis and cellular locomotion of amoebas, fungi, and animal cells (Bresnick 1999) and might play an important role in smooth muscle cells.

The myofibrils of striated muscles (skeletal and cardiac) are composed of sarcomeres in series. Both ends are demarcated by Z-lines (also known as Z-bands or Z-disks), thin disks distinguished by high protein density, high refractive index, and a high electron density. A series of bands-ordered half I-band, A-band, half I-band, which display differential light-optical and electron-optical properties based on their structural components-reside between the Z-lines (which bisect the continuous I-bands).

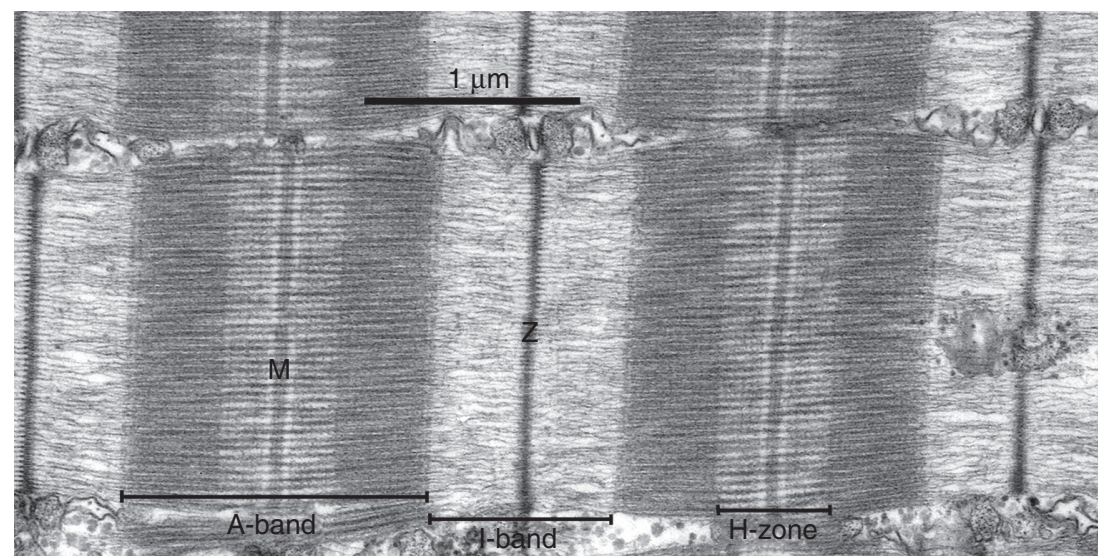

Figure 1. Organization of the sarcomere of skeletal muscle. Electron micrograph of a longitudinal thin section through a muscle fiber, with the fiber long axis horizontal. Two complete sarcomeres are shown, and elements of the sarcoplasmic reticulum separate myofibrils in the longitudinal direction. The major bands and lines are indicated, notably the thin, distinctive Z-line flanked by two low-density half I-bands, with very dense A-bands containing the thick filaments. The relative densities of the bands in this electron micrograph are related to their content of protein. The light band on either side of the M-line shows the extent of the cross-bridge-free regions of the thick filaments. The fine transverse periodicity across the A-band, at $\sim 43 \mathrm{~nm}$, is due to the periodic structure of the thick filament backbone, which in turn determines the position of relaxed cross-bridges on the surface of the filaments and is enhanced by the presence of accessory proteins. 
The A-band at the center of the sarcomere is anisotropic (A-band namesake; displaying birefringence) and electron dense as a result of the presence of parallel aligned thick filaments, primarily composed of myosin (Craig 2004). On either side of the A-band and bisected by the Z-line is the Iband, which is weakly birefringent (nearly isotropic) as a result of being largely composed of actin and its associated proteins (thin filaments). These actin filaments are thinner, more flexible, and less well aligned than the myosin filaments that occupy the A-band (Craig 2004) and span from the Z-line to the edge of the H-band (see below) of the Aband. Thus, the outer regions of the A-band are denser than the central regions, including the $\mathrm{H}$-band, because of the overlap of actin and thin and thick filaments. In threedimensional configuration, the thin and thick filaments of the sarcomere are arranged in a double-hexagonal array, where, in cross-sectional view of the overlap regions, thick filaments reside at the corners of perfect hexagons, and six thin filaments surround each thick filament, forming a second hexagonal array. This arrangement results in each thin filament being equidistant from the three adjacent thick filaments with which it interacts.

The H-band (also known as the H-zone; Fig. 1) is an area of the A-band in which the thick filaments are the only longitudinal elements; however, there are three distinct segments to this region. The M-band is the dense, very central region of the $\mathrm{H}$-band that contains cross-links that connect the thick filaments into a network. Immediately flanking the M-band are narrow lighter bands called the L-zones (also known as the bridge-free zones), which, in addition to the M-band, is a region in which no myosin cross-bridges protrude from the thick filament. The rest of the $\mathrm{H}-$ band is denser than the L-zone because of myosin crossbridges radiating from the surface of the thick filaments. Sarcomere shortening entails sliding of thin filaments past the thick filaments into the A-band, such that the I- and Hbands shorten, whereas the widths of the Z-line, A-band, $\mathrm{M}$-line, and L-zone remain unchanged. This was first described in detail by Huxley and Niedergerke and Huxley and Hanson in the 1950s (Huxley and Niedergerke 1954; Huxley and Hanson 1954), forming the basis for the "sliding filament theory" of muscle contraction.

In addition to the actin backbone, skeletal and cardiac muscle thin filaments contain the two major proteins $\mathrm{Tm}$ and $\mathrm{Tn}$ that, together, confer $\mathrm{Ca}^{2+}$ regulation to the contractile system. Together, the $\mathrm{Tn}-\mathrm{Tm}$ complex sterically blocks actin-myosin interactions in the resting muscle cells. $\mathrm{Ca}^{2+}$ released from the SR causes conformational changes in the Tn complex, allowing myosin to bind to actin. This $\mathrm{Tn}-\mathrm{Tm}$-mediated regulation of the actin-myosin interaction is known as thin filament regulation (explained in detail below).
Another major component of the sarcomere is the giant protein titin (also known as connectin; $\sim 3-3.7 \mathrm{MDa}$ ). Titin anchors into the Z-line at its amino terminus, spans across the half I-band, interacts with the thick filament at the edge of the A-band, and lies along the thick filament surface, with its carboxyl terminus at the M-line. At least six titin molecules are associated with each thick filament, three on either side of the M-line. The I-band region of titin is a stretchable region responsible for elasticity of the resting muscle. This elasticity keeps the A-band centered in the sarcomere during contraction and stretching (Horowits and Podolsky 1987) and can be fine-tuned by splice variants containing I-band-spanning regions of differential length (Granzier and Labeit 2002).

\section{THIN FILAMENTS}

Actin monomers (G-actin) polymerize into long filaments (F-actin) at physiological ionic strength and in the presence of $\mathrm{Mg}^{2+}$ and $\mathrm{K}^{+}$, forming a tightly wound helix with a pitch of $5.9 \mathrm{~nm}$ (reviewed by Pollard 2016). ATP is hydrolyzed to ADP during this process, but it is not essential for the polymerization itself. Although the length of actin filaments is not set or even stable in the absence of other regulators, length stabilization can be achieved by the addition of capping proteins that block monomer loss or addition at the ends of filaments.

An important feature of actin filament formation is intrinsic structural and functional polarity caused by the uniform orientation of actin monomers in the filament and nonidentical domains that comprise the monomers, thus, giving each end different properties. Direct evidence of this polarity was shown by fully decorating actin filaments with myosin heads, resulting in filaments seemingly covered with "arrowheads." These arrowheads point away from the Z-line and, thus, are consistent with there being a directionality of the myosin-actin interaction for sliding filaments during muscle shortening.

Within the sarcomere, actin filaments polymerize from the edges of the Z-line, with their "barbed" ends toward the Z-line end. The protein Cap Z (the muscle isoform of capping protein; also known as $\beta$-actinin) blocks the barbed ends of the actin filaments in the Z-line and might contribute to initiating their polymerization (Casella et al. 1989; Maruyama 2002), whereas members of the formin family of proteins regulate actin assembly and maintenance (Taniguchi et al. 2009). The pointed ends of the thin filaments are capped by tropomodulin and $\mathrm{Tm}$ in the sarcomere (see Fig. 2) (Gregorio et al. 1995; Almenar-Queralt et al. 1999; Rao et al. 2014). Additionally, as filament length can be well specified in different muscles, a "yardstick" is also necessary before capping, which is thought to be a role 


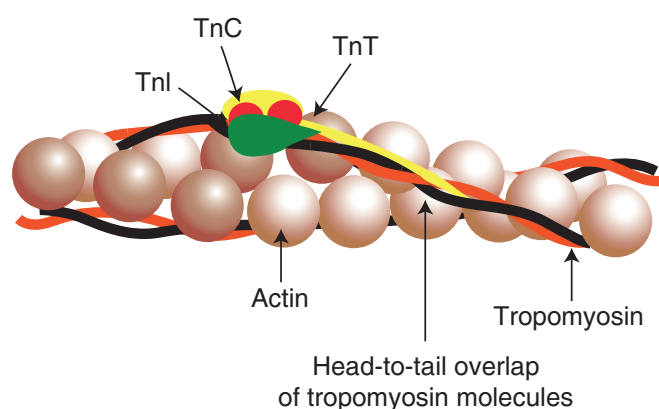

Figure 2. Regulation of thin filaments. The thin filament proteins tropomyosin and troponin regulate the contraction of striated muscle. Tropomyosin is an elongated, coiled-coil molecule that binds to the surface of the thin actin filament. Troponin comprises three subunits-troponin $\mathrm{T}(\mathrm{TnT})$, which binds to tropomyosin; troponin I (TnI), which binds to both actin and tropomyosin; and troponin C (TnC), which confers $\mathrm{Ca}^{2+}$ sensitivity to the system. The relative position of these proteins on the actin filament is schematized.

fulfilled by nebulin acting in conjunction with tropomodulin. Interestingly, live muscle fibers, especially ones subjected to prolonged periods of contractile activity, contain a population of shorter filaments, suggesting that thin filament length is dynamic, possibly the result of the cyclical occurrence of thin filament breakage and regeneration on muscle contraction (Littlefield et al. 2001).

\section{REGULATION OF STRIATED MUSCLE CONTRACTION RESIDES ON THE THIN FILAMENT}

The thin filament proteins $\mathrm{Tm}$ and $\mathrm{Tn}$ respond to $\mathrm{Ca}^{2+}$ to regulate striated muscle contraction, as shown schematically in Figure 2. Tm is a long, thin, two-stranded $\alpha$-helical coiled-coil molecule. The strands associate end-to-end to form continuous strands that occupy the two grooves of the actin helix in the native thin filament. Each Tm molecule spans seven actin subunits. In contrast, $\mathrm{Tn}$ is a complex of three subunits- $\mathrm{TnT}$, TnI, and $\mathrm{TnC}$ - one complex bound to each $\mathrm{Tm}$ molecule. The elongated $\mathrm{Tn} T$ molecule attaches the Tn complex to Tm, whereas the other two subunits are globular and link TnT to actin. The Tm-Tn repeat is longer than the pitch of the actin helix and, thus, the position of Tn has no relationship to the azimuthal orientation of actin monomers relative to thick filaments.

At low levels of free $\mathrm{Ca}^{2+}$, Tn constrains the position of $\mathrm{Tm}$ on the actin filament, and the $\mathrm{Tn}-\mathrm{Tm}$ complex sterically blocks actin-myosin interactions (Xu et al. 1999). The ability of $\mathrm{Ca}^{2+}$ to activate contraction (i.e., allow actinmyosin interaction) when released from the SR is mediated by binding to the low-affinity amino-terminal $\mathrm{Ca}^{2+}$ binding site(s) of $\mathrm{TnC}$ within the Tn complex. Note that there are two isoforms of $\mathrm{TnC}$ in striated muscle-fast $\mathrm{TnC}$ with two functional amino-terminal $\mathrm{Ca}^{2+}$ binding sites and slow/cardiac TnC with one functional site-resulting in different sensitivities to $\mathrm{Ca}^{2+}$. The binding of $\mathrm{Ca}^{2+}$ to the low-affinity binding site(s) of $\mathrm{TnC}$ releases $\mathrm{Tm}$ from its steric blocking position, allowing myosin to interact with actin and generate force. This shift is mediated through conformational changes in TnI, which are transmitted to $\mathrm{Tm}$ by TnT. Once Tm is allowed to move to its preferred position on the thin filament where it does not block myosin binding to actin, the strong binding of myosin crossbridges to actin has a synergistic effect. They help move Tm further away from the myosin binding site, thus, creating cooperativity in activation of the thin filament (Weber and Murray 1973).

In the case of cardiomyocytes, posttranslational modifications of a cardiac-specific isoform of TnI (cTnI) modify the regulation of thin filaments. For example, phosphorylation of cTnI on Ser23/24 by protein kinases PKA, PKG, $\mathrm{PKC} \beta / \delta$, or PKD1 reduces the sensitivity to $\mathrm{Ca}^{2+}$ (Layland et al. 2005; Solaro et al. 2013), thereby simultaneously raising the activation and lowering the relaxation threshold of the sarcomere. Although other $\mathrm{CTnI}$ phosphorylation sites exist, the consequences of their phosphorylation remain ambiguous-however, they might be involved in cardiomyopathies (Solaro et al. 2013).

\section{THICK FILAMENTS}

Muscle myosin II is a hexameric protein consisting of four light chains and two heavy chains (for review of myosin motor design, see Sweeney and Holzbaur 2016). The heavy chains contain two distinct regions-the "head" and the "rod." The rod is an $\alpha$-helical coiled-coil structure $\sim 1500 \AA$ long and $20 \AA$ in diameter (Huxley 1963) that connects to the two globular heads via a flexible hinge (Slayter and Lowey 1967). Interactions among myosin rods are necessary for the assembly of myosin molecules into thick filaments. The first high-resolution crystal structure of the myosin head (Rayment et al. 1993) revealed the active site for ATP hydrolysis, binding of two light chains to an extended $\alpha$-helix just before the tail domain and the binding site for actin filaments. The regions of the myosin heavy chain involved in nucleotide-, actin-, and lightchainbinding were first identified through biochemical studies.

As shown in Figure 3, the myosin molecule can be divided into individual heavy meromyosin (HMM) and light meromyosin (LMM) fragments by limited tryptic or chymotryptic digestion, in which the HMM portion of the major histocompatibility complex (MHC) $(\sim 150 \mathrm{kDa})$ contains the enzymatically functional head (S1) and amino-terminal portion (S2) of the rod. The HMM and S1 


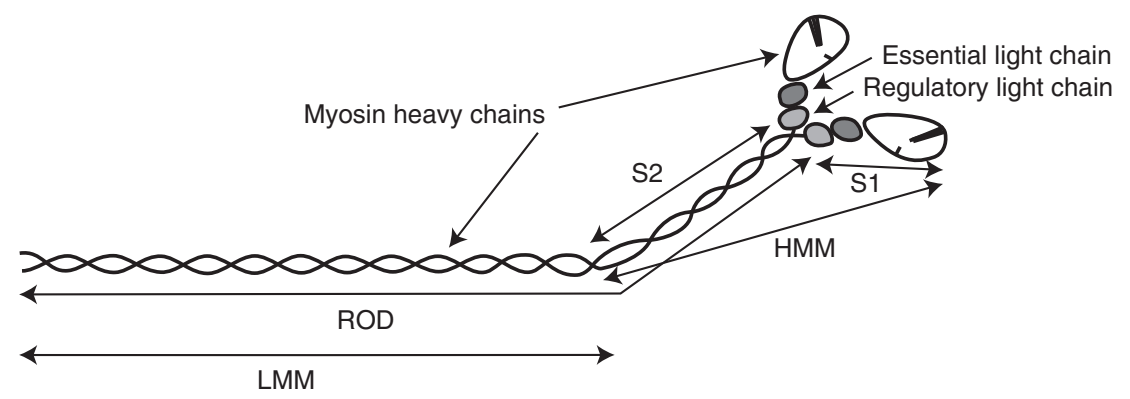

Figure 3. The myosin molecule and its proteolytic fragments. Illustrated is the myosin molecule of muscle-myosin II - and the fragments that can be generated by limited proteolysis. The catalytic portion of the molecule is in the two enzymatically functional head (S1) fragments, each containing a nucleotide-binding site and an actin-binding site. The S1 fragment is commonly referred to as the "myosin head." The remainder of the myosin molecule-the "rod"-comprising the amino-terminal portion (S2) and light meromyosin (LMM) fragments, forms an $\alpha$-helical coiled coil. LMM forms the backbone of myosin filaments. A two-headed, catalytically active fragment of myosin known as heavy meromyosin (HMM) does not form filaments, and this serves a useful purpose for solutionbiochemistry studies.

proteolytic fragments of all types of muscle myosin have been used extensively for the delineation of actin-myosin kinetics in solution as they do not aggregate at the low ionic strengths used in such assays. Each of the two myosin heads is associated with two myosin light chains, one of $\sim 18 \mathrm{kDa}$ and the other in the range of $16.5-24 \mathrm{kDa}$, making a total of four light chains per myosin molecule (Lowey and Risby 1971; Frank and Weeds 1974). These myosin light chains are known as the essential light chain (ELC), immediately distal to the myosin head, and the RLC, immediately preceding the myosin coiled-coil (Fig. 3). The RLC can be phosphorylated on Thr18/Ser19 (in humans) (Perrie et al. 1973), and this phosphorylation regulates smooth and nonmuscle myosin activity (hence the name). In striated muscle, this phosphorylation can modulate the ATPase cycle, but not turn the actin-myosin interaction on or off.

The myosin II of skeletal, cardiac, and smooth muscle polymerizes under physiological ionic conditions to form bipolar thick filaments $1.6-\mu \mathrm{m}$ long in all vertebrate skeletal muscles, regardless of muscle or species of origin. The LMM region of the tails forms the core of the filaments, overlapping in opposite directions in the bare zone in the middle of the filament. Lateral to the bare zone, the HMM regions, consisting of the S2 region of the tail and the two S1 heads, swing out on the filament surface, allowing the heads to form cross-bridges with the thin filaments. In the absence of RLC phosphorylation, the heads pack against the thick filament backbone in a manner that gives rise to extremely low ATP turnover, known as the "superrelaxed" state. In the "relaxed" state (i.e., RLC phosphorylation), the two heads of each myosin molecule either remain closely apposed to each other or assume partly independent positions, with the S2 region forming a movable link between the two heads.

\section{MYOSIN ISOFORMS OF MUSCLE}

Myosin II is the molecular motor that powers contraction of skeletal, cardiac, and smooth muscle. Its structure and function has been reviewed recently (Sweeney and Holzbaur 2016). As is particularly evident in skeletal muscle, differential expression of myosin isoforms between myocytes, and even coexpression of multiple myosin isoforms within a myocyte, provides a means to endow myocytes with a range of contractile properties. Mammalian myocytes primarily express four gene families of myosin II heavy chains, including fast skeletal, cardiac/slow skeletal, smooth, and nonmuscle. Less commonly, striated myocytes express three additional sarcomeric myosin heavy chain genes termed "superfast," "slow tonic," and "extraocular slow" (Desjardins et al. 2002; Rossi et al. 2010).

The fast skeletal myosin gene locus (on chromosome $17 \mathrm{p} 13$ in humans) comprises six distinct heavy chain genes: embryonic, perinatal (or neonatal), fast-type IIa, fast-type IIx (or IId), fast-type IIb, and extraocular (Mahdavi et al. 1986; Weiss et al. 1999). The IIb isoform is abundant in the muscles of smaller mammals, particularly rodents, but is not expressed by human myocytes. As the original type I, IIa, and IIb nomenclature was derived from histochemical characterization (Brooke and Kaiser 1970), it was unclear that the human fibers denoted IIb actually expressed the isoform later named IIx (DeNardi et al. 1993). The embryonic and perinatal isoforms are expressed during muscle development, and reexpressed in the adult during muscle regeneration from injury (Mahdavi et al. 1986). The other heavy chains are expressed in adult muscles, with the order listed above reflecting increasing speed (velocity of shortening and actin-activated ATPase activity) of the myosins that they form. In mammals, an additional myosin heavy 
chain called "superfast" is highly expressed in jaw muscles of most mammals, but not in humans, in which this gene does not code for functional protein owing to a two-base deletion/frameshift (Stedman et al. 2004). Slow-twitch (fiber type I) skeletal muscles express the same $\beta$-cardiac muscle myosin isoform as heart cells. Expression of the $\alpha$-cardiac myosin heavy chain is much less frequent in skeletal muscle, and has only been shown in muscles of the head and neck.

\section{TITIN}

Titin is a giant, stretchable protein that spans from the Z-line, through the half I-band, over the thick filament, and ends at the M-line. The extensibility of the titin molecule and, thus, the intrinsic passive tension of myofibers and cardiomyocytes, is attributed to the I-band spanning region. In skeletal muscle, this region consists of (from the amino terminus) a proximal immunoglobulin (Ig) domain, a variable number of Ig domains, an N2-A segment, a PEVK domain, and a distal series of Ig domains before reaching the edge of the A-band. Cardiac muscle contains a stiff N2-B segment between the proximal and distal regions of Ig domains, and might or might not have a complementary, more compliant, N2-A segment. Therefore, cardiac titin isoforms are termed "N2-B" (absence of N2A) or "N2-BA" (contains N2-A). The spring-like qualities of the I-band region have two phases: Low stretch forces straighten the irregular chain of Ig domains, and higher forces stretch the PEVK domain, which additionally interacts with the thin filament (Linke and Kruger 2010).

Differential splicing of the remarkably numerous 363 (human) exons of the single titin gene (TTN) produces titin isoforms of different lengths (Labeit and Kolmerer 1995; Bang et al. 2001). Most of the variable splicing is within the region of titin located in the I-band. Long isoforms are more compliant than short ones. For example, cardiac muscle compliance depends on the N2-BA:N2-B ratio, in which greater N2-A prevalence equates to less resistance to stretching. Genetic ablation of a protein subunit responsible for titin splicing results in large, incredibly compliant titin molecules in both heart and skeletal muscle (Methawasin et al. 2014). Posttranslational modifications, such as phosphorylation and oxidation, can also affect titin compliance, making immediate performance modifications to titin possible (Grutzner et al. 2009; Linke and Kruger 2010).

\section{ACTIN-MYOSIN INTERACTIONS WITHIN THE SARCOMERE}

The functional state of the muscle determines cross-bridge positioning and, therefore, the fine-structure of thick fila- ments and the entire A-band. In the relaxed muscle condition, which requires the presence of MgATP and very low $\mathrm{Ca}^{2+}$ levels, the myosin heads lie in close proximity to the surface of the thick filament, packed fairly tightly around the surface of the thick filament shaft in a configuration that has been called the superrelaxed state. The two heads of each molecule have slightly different positions and interact specifically with each other (Woodhead et al. 2005). The heads describe a helical path around the backbone of the thick filament. In skeletal muscle, three parallel helices wind around the filament. The distance between adjacent levels containing cross-bridges is $14.3 \mathrm{~nm}$, and the repeat of the helix is $\sim 43 \mathrm{~nm}$. This helical periodicity is prominent on the surface of thick filaments from sarcomeres fixed by rapid freezing, which preserves the cross-bridge disposition. This feature is also apparent in isolated thick filaments, with contrast enhanced either by negative staining or by platinum shadowing. In electron micrographs of thicker sections of relaxed sarcomeres, the A-band displays 11 visible transverse lines at $43-\mathrm{nm}$ repeat intervals. These lines arise as a result of the superimposition of the crossbridge profiles and accessory $\mathrm{C}$ and $\mathrm{H}$ proteins (Craig 2004).

During muscle contraction, the myosin heads interact with the thin filament in asynchronous concert, in which they appear to walk toward the barbed end of the filament. This is caused by rapid cross-bridge cycling (reviewed by Sweeney and Holzbaur 2016), in which the postpower stroke ADP $+\mathrm{P}_{\mathrm{i}}$ release allows rapid ATP binding, dissociation of the myosin head from actin, and reengagement with the thin filament further toward the barbed end (on ATP hydrolysis). This cycling occurring asynchronously among the myosin heads of each thick filament results in the Z-lines being pulled toward each other and thus results in shortening of the muscle.

\section{Z- AND M-LINES FUNCTION AS CROSS-LINKING REGIONS}

The Z-lines comprise a dense network of proteins that cross-link the thin actin filaments and transmit force along myofibrils of skeletal and cardiac muscle. Actin filaments are held in precise orthogonal disposition within the Z-lines, which is quite different from the hexagonal array of the A-band overlap region. Transition between the orthogonal and hexagonal dispositions is gradual at longer (i.e., resting) sarcomere lengths-however, it becomes increasingly abrupt at shorter lengths, and even problematic when the sarcomere is shortened in a way that the edges of the A-band are very close to Z-lines by hypercontraction.

Within the Z-line, the barbed end of each actin filament (each capped by Cap Z) is connected to the barbed ends of 
the four nearest thin filaments from the adjacent sarcomere by cross-links of mostly $\alpha$-actinin, a main component of Zlines, which can generate a visible zigzag pattern in simple $\mathrm{Z}$-lines. The thickness of the Z-line varies from species to species, depending on the length and disposition of connecting links. In fish, for example, the Z-line is very narrow, with the links forming a simple, woven pattern in cross sections. Frogs, in contrast, have longer connecting links that take two different configurations in cross section, depending on the sarcomeric state, resulting in a wider Z-line. Z-lines in mammalian muscle are thick owing to longitudinal overlap of thin filaments from two adjacent sarcomeres with two or more tiers of periodically disposed cross-links connecting them. Typically, fibers that sustain prolonged contractile activity, such as cardiac muscle, have Z-lines of thicker width, with more connecting tiers between the thin filaments.

The M-line is a dense complex of proteins found in the center of the A-band that links the thick filaments together within the cross-bridge-free zone and stabilizes the hexagonal array. In its most complete form, the M-line has three levels of struts that directly connect the thick filaments to each other. Some components of the M-line probably wrap tightly around the surface of the thick filaments, as thick filaments are wider in the M-line than in adjacent $\mathrm{L}$-zones.

Like the Z-line, the M-line has a complex composition that includes $M$ protein, myomesin, and creatine kinase. Myomesin, in particular, is important as a structural linker between the thick filaments and, thus, serves as the major cross-linking protein of the M-line (Lange et al. 2005). The extent of thick filament cross-linking by the M-band depends on the fiber type, as it is most extensive in fast-twitch fibers, somewhat incomplete in slow-twitch fibers, and absent in slow-tonic fibers of all vertebrates. Additionally, some mammalian extraocular muscles completely lack an M-line. Closer to the sarcolemma, both M- and Z-lines of myofibrils are peripherally associated with obscurin and ankyrin, which mediate the interaction between myofibrils and the cytoskeleton.

\section{FORCE TRANSMISSION}

Muscle contraction entails the sliding of the thin filaments past the thick filaments, resulting in shortening of the sarcomere and, thus, the entire myocyte. The resulting longitudinal force is transmitted through the extracellular matrix $(\mathrm{ECM})$ to the bone via the tendon. As individual myocytes rarely run the entire length of the muscle and are not rigidly connected to each other, the effective transmission of force from the myofilaments to the ECM is crucial for the function of the whole muscle. Cross-bridges also generate radial forces during contraction that compress the myofilament lattice (Maughan and Godt 1981). These radial forces result from the rigid connection of the thin filaments to the Z-line. As tension can change the conformation of the Z-line, the degree of compression is greater during eccentric (lengthening) than isometric contractions. Thus, myocytes have structural elements to withstand and effectively transmit both longitudinal and radial forces. Reinforcing molecules, including the dystrophin-glycoprotein complex (DGC) and integrin complexes, provide mechanical linkages from the myofilaments to the ECM.

Dystrophin, a vital force-transmission element, is a large protein with an actin-binding domain at the amino terminus, 24 spectrin repeats, and a carboxy-terminal region that associates with a complex of transmembrane glycoproteins, which comprise the dystroglycan-sarcoglycan complex. Outside the cell, $\beta$-dystroglycan binds laminin in the basal lamina surrounding the cell. These molecular interactions provide a direct connection between actin filaments and the ECM, enabling the transmission of force developed by muscle contraction, both longitudinal and radial, to the ECM. The intermediate filament protein desmin is thought to link the Z-line to dystrophin, enabling this distribution of tension. The importance of this cytoskeletal-ECM link is highlighted by the severe muscle degeneration that occurs in Duchenne muscular dystrophy (DMD), in which the gene encoding dystrophin is mutated and a functional protein is not expressed to provide this essential link.

Even with dystrophin present, the force generated by muscle contraction can injure myocytes. The extent of this damage is correlated with both the magnitude and duration of force generation and is especially prevalent in lengthening (eccentric) contractions, because the peak forces exceed the maximal isometric force of the muscle/ myocyte. Contraction-induced damage, however, can be a stimulus for myocyte hypertrophy, which can begin with $\mathrm{Ca}^{2+}$ entering cells through damaged membranes.

Given the crucial nature of the mechanical links between myofilaments and the ECM, a system of communication is needed to regulate myocyte growth, differentiation, and apoptosis (programmed cell death). A number of transmembrane proteins, including members of the integrin family, mediate this mechano/ECM-centric regulation. Integrin receptors are dimers with extracellular domains that bind to the ECM (specifically to collagen, laminin, or RGD sequences), single transmembrane domains, and cytoplasmic domains that bind to the actin cytoskeleton and associated proteins, such as vinculin, talin, and $\alpha$-actinin. Integrins use nonreceptor protein tyrosine kinases (PTKs) to transduce information from the ECM to the nucleus, thereby allowing the cell to adapt accordingly to extracellularly derived mechanical stimuli. 
Focal adhesion kinase (FAK) is an example of a PTK that plays a central role in integrin signaling and has been implicated in the regulation of myofiber growth (Fluck et al. 1999).

\section{SIGNALING ROLES OF THE SARCOMERE}

In addition to structural and force-producing roles, sarcomeres are involved in signaling processes that provide cellular feedback in response to contraction-based stimuli. As a transmitter of force generated by the actin-myosin interaction, the Z-line is an ideal location for force-sensing mechanisms enabling the myocyte to adapt to load requirements. Indeed, Z-line structural proteins with signaling potential include members of the myotilin, FATZ, and enigma protein families (von Nandelstadh et al. 2009). The myotilin family includes myotilin, palladin, and myopalladin, which all contain Ig domains and bind $\alpha$-actinin, filamin, and FATZ. The FATZ family includes three forms of FATZ (also known as casarcin and myozenin), with FATZ-1 and FATZ-3 expressed in fast-twitch muscle, and FATZ-2 in slow-twitch and cardiac muscle. FATZ-family-binding partners include myotilin, filamin, telethonin, $\alpha$-actinin, and cypher. Proteins of the enigma family contain an amino-terminal PDZ domain and 0-3 LIM domains at the carboxyl terminus. Cypher (also known as ZASP or oracle) is the most studied enigma member (Faulkner et al. 1999; Passier et al. 2000; Zhou et al. 2001) and might serve as a linker-strut by binding to $\alpha$-actinin through its PDZ domain and could be involved in signaling as a binding partner to protein kinase $\mathrm{C}$ through its LIM domains. Cypher is essential for maintaining Z-band structure and muscle integrity (Zhou et al. 2001), and mutations in cypher lead to dilated cardiomyopathy and skeletal muscle myopathies that are termed "zaspopathies."

Titin is another major signaling regulator of the sarcomere. In addition to having its own intrinsic enzymatic activity in the mechanosensing titin-kinase domain near the M-line, titin contains many binding sites for key regulators of mechanosensing, myocyte hypertrophy, and protein turnover. These titin ligands include the mechanosensing tandem muscle LIM protein (MLP) and telethonin at the Z1/Z2 domain (located at the Z-line); calpain proteases and muscle ankyrin repeat proteins (MARPs), which are involved in titin performance modification and transcriptional regulation, in the I-band region; and the E3 ubiquitin ligases, MuRFs, which mediate atrophy, toward the M-line. Although the precise role of each member is not fully understood, having these regulatory systems integrated into this giant, elastic protein likely provides the most rapid response to mechanical stimuli experienced by the sarcomere.

\section{DIFFERENCES BETWEEN CARDIAC AND SKELETAL MUSCLE}

Although cardiac myocytes are striated with sarcomeres of very similar composition to those of skeletal muscle, there are important structural and functional differences between the two muscle types. The most obvious difference is the size of the cells. Whereas skeletal muscle cells are multinucleated giants, cardiomyocytes are branched cylinders, typically $100-300 \mu \mathrm{m}$ in length and $20-30 \mu \mathrm{m}$ in diameter, although they can undergo hypertrophy to as large as $150-200 \mu \mathrm{m}$ in diameter, with one, two, or three nuclei (most are binucleated).

Cardiac and skeletal muscle cells also differ in their electrical properties. Skeletal muscle cells are electrically isolated from each other, whereas all of the cells of the heart form an electrical syncytium. Cardiac cells are coupled electrically by gap junctions located adjacent to specialized mechanical connections at the ends of the cells called intercalated disks. Although the skeletal muscle cell action potential is similar to that of nerve cells, both in time course and mechanism, the cardiac action potential is long in duration $(200-300 \mathrm{msec})$ and complex in mechanism. $\mathrm{Ca}^{2+}$ influx is one component of the cardiac action potential. Thus, skeletal muscle cells rely entirely on SR as the source of activator $\mathrm{Ca}^{2+}$ ions, whereas cardiac cells derive activator $\mathrm{Ca}^{2+}$ ions from both the SR and extracellular pools. In addition, the influx of extracellular $\mathrm{Ca}^{2+}$ through voltage-gated $\mathrm{Ca}^{2+}$ channels triggers the release of $\mathrm{Ca}^{2+}$ from the SR in mammalian hearts, a process known as calcium-induced calcium release (CICR) (Bers 2002).

Vertebrate cardiac muscle cells express two different myosin heavy chain isoforms. These two cardiac myosin heavy chain genes ( $\alpha$ and $\beta$ ) are found in tandem on human chromosome 14q12 corresponding to Myh6 and Myh7 genes (Mahdavi et al. 1984). In adult human, the primary isoform is $\beta$, whereas in the mouse it is $\alpha$ (Lompre et al. 1981). In cardiac muscle, coexpression of the two heavy chains leads to three myosin forms, known as V1, $\mathrm{V} 2$, and V3 (listed in order of decreasing enzymatic activity), in which V1 is an $\alpha-\alpha$ homodimer, V3 is a $\beta-\beta$ homodimer, and V2 is a heterodimer between $\alpha$ and $\beta$. The $\alpha$ form has a higher ATPase activity than the $\beta$ (Pope et al. 1980), and is primarily expressed in humans as a fetal gene or in pathological conditions (although the opposite is true in rodents). Cardiac muscle cells share a number of contractile protein isoforms with slow skeletal muscle, including a $\beta$-cardiac muscle myosin isoform.

As in skeletal muscle, $\mathrm{Ca}^{2+}$ binding to Tn $\mathrm{C}$ regulates the actin-myosin interaction in cardiac muscle. Cardiomyocytes, however, have evolved mechanisms to alter thin filament regulation to adapt the contractility properties 
rapidly in response to immediate stimuli. This is important because every cardiac cell contracts with each beat of the heart, so any change in the force produced by the heart must be accomplished by modifying the force output of the individual cells. The heart has the ability to change its output, and the rate at which it performs work on a beat-tobeat basis. Additionally, in response to chronically altered conditions, such as hypertension or damage due to coronary occlusion, or repeated acute stimuli, as in exercise training, the heart adapts over many days by remodeling its cellular constituents.

There are two major classes of these thin filament regulatory mechanisms, which allow cardiac muscle cells to alter their force response to a given cytosolic $\mathrm{Ca}^{2+}$ concentration-length dependence of $\mathrm{Ca}^{2+}$ sensitivity and neuroendocrine control. Length dependence of calcium sensitivity, as shown with permeabilized cardiac cells, entails increasing force generation by submaximal $\mathrm{Ca}^{2+}$ stimulation with increasing sarcomere lengths (Kentish et al. 1986). Although the maximum $\mathrm{Ca}^{2+}$-activated force does not change, the threshold concentration for activation does change, altering the relationship between force and $\mathrm{Ca}^{2+}$ concentration. The basis for the mechanism is ambiguous, as a length-dependent change in $\mathrm{Ca}^{2+}$ binding to Tn C is not dependent on the cardiac isoform of Tn C (Moss et al. 1991), although some evidence suggests that titin is involved in this phenomenon (Le Guennec et al. 2000). This mechanism should contribute to the lengthtension (cellular level) or pressure-volume (ventricular level) relationships.

The neuroendocrine system also affects thin filament control, allowing a change in contractile properties in response to $\mathrm{Ca}^{2+}$. Specifically, $\beta$-adrenergic receptor activation by norepinephrine (sympathetic nervous system) or epinephrine (endocrine system) results in elevation of cyclic AMP (cAMP) by means of adenylyl cyclase and subsequent activation of the Ser/Thr-protein kinase PKA. The cardiac-specific isoform of TnI (cTnI) is a phosphorylation target of PKA, and thus $\beta$-adrenergic stimulation results in the reversible phosphorylation of cTnI. Phosphorylation of cTnI decreases the affinity of $\mathrm{TnC}$ for $\mathrm{Ca}^{2+}$, resulting in faster dissociation of $\mathrm{Ca}^{2+}$. This increases the rate that the muscle relaxes and, thus, a higher $\mathrm{Ca}^{2+}$ concentration (up to a fivefold change) is necessary to produce a given $\mathrm{Ca}^{2+}$-induced force.

The physiological consequence of the decrease in $\mathrm{Ca}^{2+}$ sensitivity during $\beta$-adrenergic stimulation is an important feature of cardiac physiology. $\beta$-adrenergic stimulation increases the heart rate, and faster relaxation of the ventricles allows more time for them to fill. Thus, manipulation of Tn contributes toward $\beta$-adrenergic-induced increases in cardiac output. $\beta$-adrenergic stimulation of the heart and activation of PKA also results in phosphorylation of phospholamban, a subunit of the SR $\mathrm{Ca}^{2+}$ pump SERCA. Phosphorylated phospholamban does not inhibit the pump, which allows faster removal of $\mathrm{Ca}^{2+}$ from the cytosol into the SR (Wegener et al. 1989). This further speeds relaxation, but also results in loading of the SR with more $\mathrm{Ca}^{2+}$, thus, more $\mathrm{Ca}^{2+}$ is available for release despite the decreased systolic interval.

Phosphorylation of the RLC also affects cardiac muscle contraction. RLC phosphorylation increases both the amount of force produced at a given submaximal $\mathrm{Ca}^{2+}$ concentration and the rate at which cross-bridges produce force (Davis et al. 2001). Both alterations are a result of the phosphorylated myosin cross-bridges entering the forceproducing state(s) more rapidly than dephosphorylated cross-bridges. The cascade of events leading to myosin phosphorylation is the same as in smooth muscle- $\mathrm{Ca}^{2+}$ binds to calmodulin, which activates myosin light-chain kinase (MLCK). The myosin light-chain phosphatase dephosphorylates RLC, but its regulation in striated muscle is not well understood.

Finally, note that the term "contractility" is used to define the ability of heart muscle to develop force at a fixed length (or pressure at fixed ventricular volume). However, a variety of correlative physiological parameters based on the properties of muscle (i.e., rate of rise of force, maximal velocity of shortening) are also used to define contractility and are hotly debated. Factors that influence contractility are termed "inotropic" agents. Thus, increasing frequency of contraction and sympathetic stimulation are "positive inotropic" factors. "Negative inotropic" factors include certain diseases that lead to heart failure and some drugs (e.g., barbiturates) that decrease the strength of the heart. "Lucitropy" refers to the increase in the rate of cardiac relaxation.

\section{DIFFERENCES BETWEEN STRIATED AND SMOOTH MUSCLE}

The most notable functional requirement of the skeletal musculature is the ability to provide rapid bursts of force generation and/or movement under the control of the nervous system. Not only must the movement itself be rapid, but contractile activity must turn on and off equally rapidly. Cardiac muscle requires similar circumstances to maintain proper blood circulation and perfusion based on an organism's energy requirements. Smooth muscles, in contrast, subserve functions in the body that are more or less continuously ongoing, frequently involving (as in the case of vasoconstriction to maintain arterial pressure or direct blood flow) very little motion but, instead, the maintenance of steady tonic forces. In those smooth muscles in 
which motion is important for function (e.g., intestinal peristalsis), the movements can be quite large with respect to muscle size but are typically several-hundred-fold slower than in skeletal muscles. To fulfil these distinct functional roles, the striated and smooth muscles have evolved distinct structural, physiological, and biochemical profiles.

Smooth muscle cells are arranged in layers around tubular and hollow organs. For the vasculature, smooth muscle is arranged in two individual layers-an inner circular layer of smooth muscle and an outer longitudinal layer of smooth muscle. For arteries, the circular layer is much thicker than the longitudinal layer, owing to the fact that the artery must contract to increase vascular resistance against arterial pressure. For venous tissue, the circular layer is less developed as the venous pressure is much lower. For the hollow organs, such as the urinary bladder, which must undergo large volume changes, smooth muscle layers are organized into irregular sheets so that, during contraction, the organ will decrease in size, much like letting air out of a balloon. For the gastrointestinal system, the outer longitudinal layer of smooth muscle is more developed to propel a food bolus down the alimentary canal.

One of the immediately evident differences between skeletal/cardiac muscle cells and smooth muscle cells is in their size and general appearance. Smooth muscle cells are among the smallest cells in the body, typically spindleshaped fibers $50-200-\mu \mathrm{m}$ long, with a broadest diameter of $2-8 \mu \mathrm{m}$. The absence of striations (and, hence, the name) is due to the absence of the high degree of order that characterizes the contractile filament lattice of skeletal/ cardiac muscle. Whereas smooth muscle cells contain $\sim 75 \%$ of the total amount of contractile protein (actin plus myosin plus Tm) as other muscle tissues, the distribu- tion of protein types is strikingly different, as depicted in Figure 4.

Smooth muscle actin comprises the thin filament lattice, which can be as much as twice the concentration in smooth muscle as compared with skeletal muscle actin in skeletal muscle, relative to myosin content (Gabella 1984). The thin filaments are dispersed richly throughout the cell, oriented generally parallel to the longitudinal axis of the cell, sometimes appearing clustered. Thin filament length is on average longer than found in skeletal muscle. Some of the actin filaments are anchored to "dense bands" on the plasma membrane, and others terminate in cytoplasmic "dense bodies" (Fig. 4). A network of intermediate filaments composed of desmin (sometimes replaced by vimentin in some smooth muscle cells) interconnects membrane and cytoplasmic dense bodies (Stromer and Bendayan 1988) and reinforces the thin filament system. Both types of smooth muscle dense bodies contain $\alpha$-actinin. These similarities in protein content and function support the notion that the intermediate filament lattice/ dense body network is essentially a dispersed Z-line-or, more likely, Z-lines arose as an aggregate of dense bodies during the evolution of striated muscle.

$\mathrm{Tm}$ is present in smooth muscle in about the same ratio to actin as in skeletal muscle, and is similarly associated with actin filaments. Smooth muscle, however, lacks Tn proteins, which are necessary to confer thin filament regulation in skeletal and cardiac muscle. Instead, the thin filaments of smooth muscle can contain either caldesmon or calponin, which appear to compete for occupancy on thin filaments (Makuch et al. 1991). Although the exact physiological roles of these two proteins in smooth muscle have not yet been established, they appear to play modula-
A

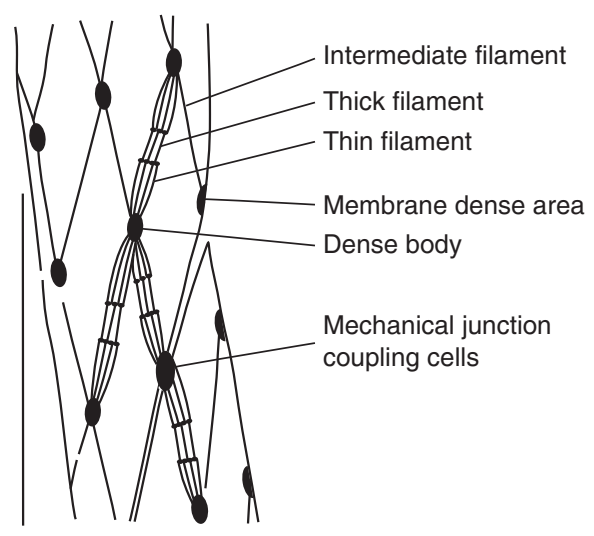

B

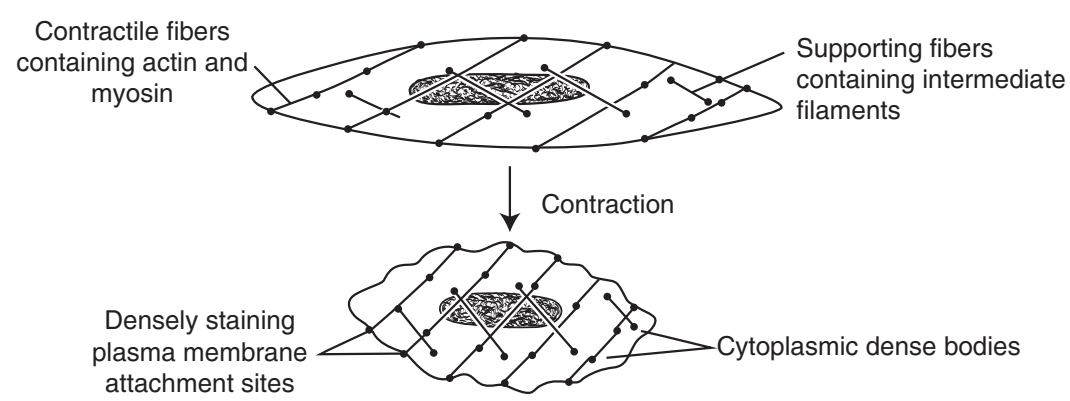

Figure 4. The contractile apparatus of smooth muscle. (A) Schematic of the key components of the force-generating protein network in mammalian smooth muscle. $(B)$ The organization and rearrangements of the smooth muscle cell cytoskeleton during contraction. 
tory functions in fine-tuning the smooth muscle contractile characteristics.

The smooth muscle myosin molecule is morphologically identical to skeletal muscle myosin, but biochemically quite different, especially with regard to the regulation of the actin-activated ATPase activity. Smooth muscle myosin content is very low, being only about $20 \%$ relative to that of skeletal muscle. The smooth myosin forms large, side-polar filaments, which appear to be made up of assemblies of bipolar mini-filaments. A fraction of the myosin content of smooth muscle can, at any given moment, be depolymerized, rather than assembled in a thick filament. This greater lability of smooth muscle myosin filaments could play a role in normal physiological processes and allow rapid restructuring of the contractile apparatus, which does not occur in the sarcomeres of striated muscles.

The lower myosin content and incomplete polymerization of myosin leads to a much lower density of smooth muscle thick filaments than in skeletal and cardiac muscle. The smooth muscle thick filaments are more or less uniformly dispersed throughout the cell, approximately parallel to the longitudinal axis. The elevated actin content leads to a final ratio of thick to thin filaments of approximately 1:15 in smooth muscle, as opposed to 1:2 in skeletal and cardiac muscle (Gabella 1984).

Although smooth muscle lacks the organized sarcomeric structure of skeletal and cardiac muscle, it is organized in contractile filament domains, containing the dense body and intermediate filament networks. The forces generated by actin and myosin interaction are transmitted to and across the cell membrane, and the contractile units in adjacent cells are linked between membrane-attachment plaques to transmit the force of contraction across cells. Because of longer thin filaments and the less constrained organization of the contractile units, individual cells can shorten by $>70 \%$ of their resting length. The thin-thick filament interaction can be termed "plastic," as the individual thin filaments are not bound by a sarcomere to associate only with a single thick filament. This plasticity has important implications for the force-length relationship, discussed in more detail below.

The mechanism of actin-activated myosin ATPase of smooth muscle is the same as striated muscle myosin, or for any myosin (discussed above, and reviewed by Sweeney and Holzbaur 2016). ATP is hydrolyzed while actin and myosin are detached from one another. Myosin cross-bridges attach to actin and then convert the chemical energy of ATP hydrolysis into mechanical energy during the sequential release of the hydrolysis products-phosphate and ADP.

$\mathrm{Ca}^{2+}$ regulates contraction of both striated and smooth muscles, but the mechanisms are different. Rather than binding to Tn on thin filaments in striated muscle, $\mathrm{Ca}^{2+}$ in smooth muscle leads to phosphorylation of the myosin RLC. Striated muscle myosin is essentially unregulated, but the myosin II of smooth muscle requires phosphorylation of RLC for ATPase activity, as does nonmuscle myosin II (Sellers 1991). Given the high concentration of myosin in smooth muscle cells, much of the myosin is polymerized into filaments even in the absence of RLC phosphorylation. However, the heads fold back on the filament backbone, leading to inhibition of ATPase activity unless there is RLC phosphorylation. Phosphorylation of either RLC allows association with actin to activate its ATPase cycle.

Like that of cardiac muscle, smooth muscle contraction is regulated by both extracellular and intracellular (SR) $\mathrm{Ca}^{2+}$ stores, which can be activated by mechanical stretch or by G-protein-coupled receptors (GPCRs; e.g., $\alpha 1$-adrenergic receptors of vascular smooth muscle). The rise of intracellular $\left[\mathrm{Ca}^{2+}\right]$, by either stimulus, targets MLCK. At low calcium concentrations, MLCK is inactive, and, as the $\mathrm{Ca}^{2+}$ concentration increases and calcium ions bind to calmodulin (which is homologous to $\mathrm{TnC}$ ), calmodulin activates MLCK, resulting in phosphorylation of RLC and enabling the myosin interaction with actin (Means et al. 1991). Although many isoforms of MLCK exist in by all types of cells, the only known substrate is RLC. MLC phosphatase dephosphorylates RLC, turning off the myosin ATPase cycle as $\mathrm{Ca}^{2+}$ levels decrease. Coordinated smooth muscle contraction or relaxation can occur through regulation of RLC phosphorylation by these two enzymes. Owing to this kinase-phosphatase-dependent regulation of contraction, smooth muscle experiences, relative to striated muscle, a slow onset and cessation of contraction and, thus, might contract long after the initial stimulus has been removed.

\section{CONCLUSION}

Table 1 summarizes the features of the three distinct classes of mammalian muscle cells. The two types of striated muscle-skeletal and cardiac-organize their contractile filaments of actin and myosin into regular arrays called sarcomeres. These striated muscles are regulated by $\mathrm{Ca}^{2+}$ binding to $\mathrm{Tn}$, which then frees $\mathrm{Tm}$ to move on actin and uncover the myosin-binding site. Smooth muscle also contracts by interaction of actin and myosin filaments, but these filaments are not in regular repeating arrays. Phosphorylation of the RLC of myosin controls the actin-myosin interaction in smooth muscle.

Despite the wealth of data amassed on all types of muscle, we still poorly understand the dynamic control of the tissues, especially how the signals associated with contraction feedback to control growth and remodeling of the 
H.L. Sweeney and D.W. Hammers

Table 1. Comparison of the properties of the three types of mammalian muscle

\begin{tabular}{llll}
\hline Characteristic & Skeletal muscle & Cardiac muscle & Smooth muscle \\
\hline Striation & Yes & Yes & No \\
Number of nuclei/cell & Many & 1,2 or 3 & 1 \\
Innervation & Somatic & ANS & ANS \\
T-tubules & Yes & Yes & No \\
SR & +++ & ++ & + to ++ \\
Regulatin of actin-myosin interaction & $\mathrm{Ca}^{2+}$ binding & $\mathrm{Ca}^{2+}$ binding & Myosin (RLC) \\
& to Tn & to Tn & phosphorylation \\
& & & (Ca ${ }^{2+}$ CaM-mediated) \\
Sources of activator Ca & SR and ECF & Tn C & MLCK \\
Site of Ca ${ }^{2+}$ action & SR & Slow & Very slow \\
Speed of contraction & Tn C & No & Yes \\
Inherent tone-low levels of maintained tension & Now to fast & Yes & Yes (phasic) \\
Physiological pacemaker potentials & No & Excitation and inhibition & Excitation and inhibition \\
Effects of nervous stimulation & Excitation &
\end{tabular}

ANS, autonomic nervous system; CaM, calmodulin; ECF, extracellular fluid; RLC, regulatory light chain; Tn, troponin; MLCK, myosin light-chain kinase; SR, sarcoplasmic reticulum.

tissues. This is a crucial area for future research as the development of therapies for cardiomyopathies, vascular diseases, the muscular dystrophies, and muscle dysfunction associated with aging all require a greater understanding of the fundamental control of the growth and remodeling processes of the muscular tissues than we currently possess.

\section{REFERENCES}

${ }^{*}$ Reference is also in this subject collection.

Almenar-Queralt A, Lee A, Conley CA, Ribas de Pouplana L, Fowler VM. 1999. Identification of a novel tropomodulin isoform, skeletal tropomodulin, that caps actin filament pointed ends in fast skeletal muscle. $J$ Biol Chem 274: 28466-28475.

Bang ML, Centner T, Fornoff F, Geach AJ, Gotthardt M, McNabb M, Witt CC, Labeit D, Gregorio CC, Granzier H, et al. 2001. The complete gene sequence of titin, expression of an unusual approximately $700-\mathrm{kDa}$ titin isoform, and its interaction with obscurin identify a novel Z-line to I-band linking system. Circ Res 89: 1065-1072.

Bers DM. 2002. Cardiac excitation-contraction coupling. Nature 415: $198-205$.

Bresnick AR. 1999. Molecular mechanisms of nonmuscle myosin-II regulation. Curr Opin Cell Biol 11: 26-33.

Brooke MH, Kaiser KK. 1970. Muscle fiber types: How many and what kind? Arch Neurol 23: 369-379.

Casella JF, Casella SJ, Hollands JA, Caldwell JE, Cooper JA. 1989. Isolation and characterization of cDNA encoding the $\alpha$ subunit of Cap Z(36/ 32 ), an actin-capping protein from the $\mathrm{Z}$ line of skeletal muscle. Proc Natl Acad Sci 86: 5800-5804.

Craig RW., ed. 2004. Molecular structure of the sarcomere. McGraw-Hill, New York.

Davis JS, Hassanzadeh S, Winitsky S, Lin H, Satorius C, Vemuri R, Aletras AH, Wen H, Epstein ND. 2001. The overall pattern of cardiac contraction depends on a spatial gradient of myosin regulatory light chain phosphorylation. Cell 107: 631-641.

DeNardi C, Ausoni S, Moretti P, Gorza L, Velleca M, Buckingham M, Schiaffino S. 1993. Type 2X-myosin heavy chain is coded by a muscle fiber type-specific and developmentally regulated gene. J Cell Biol 123: $823-835$
Desjardins PR, Burkman JM, Shrager JB, Allmond LA, Stedman HH. 2002. Evolutionary implications of three novel members of the human sarcomeric myosin heavy chain gene family. Mol Biol Evol 19: 375393.

Faulkner G, Pallavicini A, Formentin E, Comelli A, Ievolella C, Trevisan S, Bortoletto G, Scannapieco P, Salamon M, Mouly V, et al. 1999. ZASP: A new Z-band alternatively spliced PDZ-motif protein. J Cell Biol 146: $465-475$.

Fluck M, Carson JA, Gordon SE, Ziemiecki A, Booth FW. 1999. Focal adhesion proteins FAK and paxillin increase in hypertrophied skeletal muscle. Am J Physiol 277: C152-C162.

Frank G, Weeds AG. 1974. The amino-acid sequence of the alkali light chains of rabbit skeletal-muscle myosin. Eur J Biochem 44: 317-334.

Gabella G. 1984. Structural apparatus for force transmission in smooth muscles. Physiol Rev 64: 455-477.

Granzier H, Labeit S. 2002. Cardiac titin: An adjustable multi-functional spring. J Physiol 541: 335-342.

Gregorio CC, Weber A, Bondad M, Pennise CR, Fowler VM. 1995. Requirement of pointed-end capping by tropomodulin to maintain actin filament length in embryonic chick cardiac myocytes. Nature 377: 83-86.

Grutzner A, Garcia-Manyes S, Kotter S, Badilla CL, Fernandez JM, Linke WA. 2009. Modulation of titin-based stiffness by disulfide bonding in the cardiac titin N2-B unique sequence. Biophys J 97: 825-834.

Horowits R, Podolsky RJ. 1987. The positional stability of thick filaments in activated skeletal muscle depends on sarcomere length: Evidence for the role of titin filaments. J Cell Biol 105: 2217-2223.

Huxley HE. 1963. Electron microscope studies on the structure of natural and synthetic protein filaments from striated muscle. J Mol Biol 7: 281-308.

Huxley H, Hanson J. 1954. Changes in the cross-striations of muscle during contraction and stretch and their structural interpretation. Nature 173: 973-976.

Huxley AF, Niedergerke R. 1954. Structural changes in muscle during contraction; interference microscopy of living muscle fibres. Nature 173: $971-973$.

Kentish JC, ter Keurs HE, Ricciardi L, Bucx JJ, Noble MI. 1986. Comparison between the sarcomere length-force relations of intact and skinned trabeculae from rat right ventricle. Influence of calcium concentrations on these relations. Circ Res 58: 755-768.

Labeit S, Kolmerer B. 1995. Titins: Giant proteins in charge of muscle ultrastructure and elasticity. Science 270: 293-296.

Lange S, Himmel M, Auerbach D, Agarkova I, Hayess K, Furst DO, Perriard JC, Ehler E. 2005. Dimerisation of myomesin: Implications for the structure of the sarcomeric M-band. J Mol Biol 345: 289-298. 
Layland J, Solaro RJ, Shah AM. 2005. Regulation of cardiac contractile function by troponin I phosphorylation. Cardiovasc Res 66: 12-21.

Le Guennec JY, Cazorla O, Lacampagne A, Vassort G. 2000. Is titin the length sensor in cardiac muscle? Physiological and physiopathological perspectives. Adv Exp Med Biol 481: 337-348; discussion 348-351.

Linke WA, Kruger M. 2010. The giant protein titin as an integrator of myocyte signaling pathways. Physiology 25: 186-198.

Littlefield R, Almenar-Queralt A, Fowler VM. 2001. Actin dynamics at pointed ends regulates thin filament length in striated muscle. Nat Cell Biol 3: 544-551.

Lompre AM, Mercadier JJ, Wisnewsky C, Bouveret P, Pantaloni C, D'Albis A, Schwartz K. 1981. Species- and age-dependent changes in the relative amounts of cardiac myosin isoenzymes in mammals. Dev Biol 84: $286-290$.

Lowey S, Risby D. 1971. Light chains from fast and slow muscle myosins. Nature 234: 81-85.

Mahdavi V, Chambers AP, Nadal-Ginard B. 1984. Cardiac $\alpha$ - and $\beta$ myosin heavy chain genes are organized in tandem. Proc Natl Acad Sci 81: 2626-2630.

Mahdavi V, Strehler EE, Periasamy M, Wieczorek DF, Izumo S, NadalGinard B. 1986. Sarcomeric myosin heavy chain gene family: Organization and pattern of expression. Med Sci Sports Exerc 18: 299-308.

Makuch R, Birukov K, Shirinsky V, Dabrowska R. 1991. Functional interrelationship between calponin and caldesmon. Biochem J 280: $33-38$.

Maruyama K. 2002. $\beta$-Actinin, Cap Z, Connectin and Titin: What's in a name? Trends Biochem Sci 27: 264-266.

Maughan DW, Godt RE. 1981. Radial forces within muscle fibers in rigor. J Gen Physiol 77: 49-64.

Means AR, Bagchi IC, VanBerkum MF, Kemp BE. 1991. Regulation of smooth muscle myosin light chain kinase by calmodulin. Adv Exp Med Biol 304: 11-24.

Methawasin M, Hutchinson KR, Lee EJ, Smith JE 3rd, Saripalli C, Hidalgo CG, Ottenheijm CA, Granzier H. 2014. Experimentally increasing titin compliance in a novel mouse model attenuates the Frank-Starling mechanism but has a beneficial effect on diastole. Circulation 129: 1924-1936.

Moss RL, Nwoye LO, Greaser ML. 1991. Substitution of cardiac troponin $\mathrm{C}$ into rabbit muscle does not alter the length dependence of $\mathrm{Ca}^{2+}$ sensitivity of tension. J Physiol 440: 273-289.

Odronitz F, Kollmar M. 2007. Drawing the tree of eukaryotic life based on the analysis of 2,269 manually annotated myosins from 328 species. Genome Biol 8: R196.

Passier R, Richardson JA, Olson EN. 2000. Oracle, a novel PDZ-LIM domain protein expressed in heart and skeletal muscle. Mech Dev 92: 277-284.

Perrie WT, Smillie LB, Perry SB. 1973. A phosphorylated light-chain component of myosin from skeletal muscle. Biochem J 135: 151-164.

* Pollard TD. 2016. Actin and actin-binding proteins. Cold Spring Harb Perspect Biol 8: a018226.

Pope B, Hoh JF, Weeds A. 1980. The ATPase activities of rat cardiac myosin isoenzymes. FEBS Lett 118: 205-208.
Rao JN, Madasu Y, Dominguez R. 2014. Mechanism of actin filament pointed-end capping by tropomodulin. Science 345: 463-467.

Rayment I, Holden HM, Whittaker M, Yohn CB, Lorenz M, Holmes KC, Milligan RA. 1993. Structure of the actin-myosin complex and its implications for muscle contraction. Science 261: 58-65.

Rossi AC, Mammucari C, Argentini C, Reggiani C, Schiaffino S. 2010. Two novel/ancient myosins in mammalian skeletal muscles: MYH14/ $7 \mathrm{~b}$ and MYH15 are expressed in extraocular muscles and muscle spindles. J Physiol 588: 353-364.

Sellers JR. 1991. Regulation of cytoplasmic and smooth muscle myosin. Curr Opin Cell Biol 3: 98-104.

Slayter HS, Lowey S. 1967. Substructure of the myosin molecule as visualized by electron microscopy. Proc Natl Acad Sci 58: 1611-1618.

Solaro RJ, Henze M, Kobayashi T. 2013. Integration of troponin I phosphorylation with cardiac regulatory networks. Circ Res 112: 355-366.

Stedman HH, Kozyak BW, Nelson A, Thesier DM, Su LT, Low DW, Bridges CR, Shrager JB, Minugh-Purvis N, Mitchell MA. 2004. Myosin gene mutation correlates with anatomical changes in the human lineage. Nature 428: 415-418.

Stromer MH, Bendayan M. 1988. Arrangement of desmin intermediate filaments in smooth muscle cells as shown by high-resolution immunocytochemistry. Cell Motil Cytoskeleton 11: 117-125.

* Sweeney HL, Holzbaur E. 2016. Motor proteins. Cold Spring Harb Perspect Biol 10.1101/a021931.

Taniguchi K, Takeya R, Suetsugu S, Kan OM, Narusawa M, Shiose A, Tominaga R, Sumimoto H. 2009. Mammalian formin Fhod3 regulates actin assembly and sarcomere organization in striated muscles. J Biol Chem 284: 29873-29881.

von Nandelstadh P, Ismail M, Gardin C, Suila H, Zara I, Belgrano A, Valle G, Carpen O, Faulkner G. 2009. A class III PDZ binding motif in the myotilin and FATZ families binds enigma family proteins: A common link for Z-disc myopathies. Mol Cell Biol 29: 822-834.

Weber A, Murray JM. 1973. Molecular control mechanisms in muscle contraction. Physiol Rev 53: 612-673.

Wegener AD, Simmerman HK, Lindemann JP, Jones LR. 1989. Phospholamban phosphorylation in intact ventricles. Phosphorylation of serine 16 and threonine 17 in response to $\beta$-adrenergic stimulation. J Biol Chem 264: 11468-11474.

Weiss A, McDonough D, Wertman B, Acakpo-Satchivi L, Montgomery K, Kucherlapati R, Leinwand L, Krauter K. 1999. Organization of human and mouse skeletal myosin heavy chain gene clusters is highly conserved. Proc Natl Acad Sci 96: 2958-2963.

Woodhead JL, Zhao FQ, Craig R, Egelman EH, Alamo L, Padron R. 2005. Atomic model of a myosin filament in the relaxed state. Nature 436: $1195-1199$

Xu C, Craig R, Tobacman L, Horowitz R, Lehman W. 1999. Tropomyosin positions in regulated thin filaments revealed by cryoelectron microscopy. Biophys J 77: 985-992.

Zhou Q, Chu PH, Huang C, Cheng CF, Martone ME, Knoll G, Shelton GD, Evans S, Chen J. 2001. Ablation of Cypher, a PDZ-LIM domain Z-line protein, causes a severe form of congenital myopathy. J Cell Biol 155: $605-612$. 


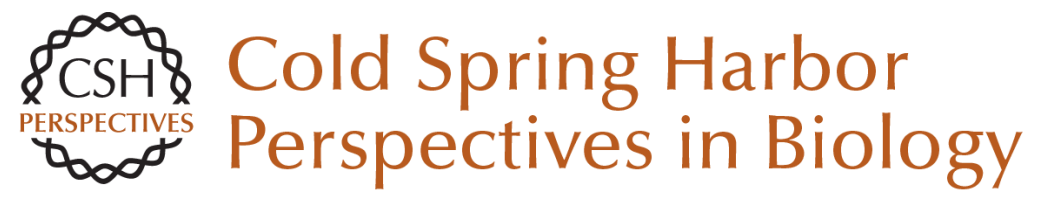

\section{Muscle Contraction}

H. Lee Sweeney and David W. Hammers

Cold Spring Harb Perspect Biol 2018; doi: 10.1101/cshperspect.a023200

Subject Collection The Cytoskeleton

Microtubules and Microtubule-Associated

Proteins

Holly V. Goodson and Erin M. Jonasson

Motor Proteins

H. Lee Sweeney and Erika L.F. Holzbaur

Myosin-Driven Intracellular Transport Margaret A. Titus

The Actin Cytoskeleton and Actin-Based Motility Tatyana Svitkina

\section{Mechanical Properties of the Cytoskeleton and Cells \\ Adrian F. Pegoraro, Paul Janmey and David A. Weitz}

Intermediate Filaments and the Regulation of Cell Motility during Regeneration and Wound Healing Fang Cheng and John E. Eriksson

Intermediate Filaments and the Plasma Membrane Jonathan C.R. Jones, Chen Yuan Kam, Robert M. Harmon, et al.

Intracellular Motility of Intermediate Filaments Rudolf E. Leube, Marcin Moch and Reinhard Windoffer

\author{
Overview of the Cytoskeleton from an \\ Evolutionary Perspective \\ Thomas D. Pollard and Robert D. Goldman
Types I and II Keratin Intermediate Filaments Justin T. Jacob, Pierre A. Coulombe, Raymond \\ Kwan, et al. \\ Muscle Contraction \\ H. Lee Sweeney and David W. Hammers \\ Type III Intermediate Filaments Desmin, Glial \\ Fibrillary Acidic Protein (GFAP), Vimentin, and \\ Peripherin \\ Elly M. Hol and Yassemi Capetanaki \\ Cytokinesis in Metazoa and Fungi \\ Michael Glotzer
}

\section{Ciliary Motility: Regulation of Axonemal Dynein} Motors

Rasagnya Viswanadha, Winfield S. Sale and Mary

E. Porter

Actin-Based Adhesion Modules Mediate Cell Interactions with the Extracellular Matrix and Neighboring Cells

Alexia I. Bachir, Alan Rick Horwitz, W. James Nelson, et al.

Microtubule-Based Transport and the Distribution, Tethering, and Organization of Organelles Kari Barlan and Vladimir I. Gelfand

For additional articles in this collection, see http://cshperspectives.cshlp.org/cgi/collection/

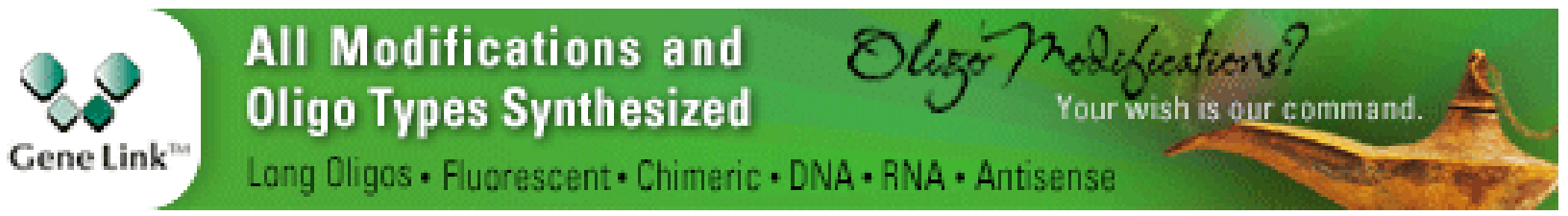

Copyright @ 2018 Cold Spring Harbor Laboratory Press; all rights reserved 
For additional articles in this collection, see http://cshperspectives.cshlp.org/cgi/collection/

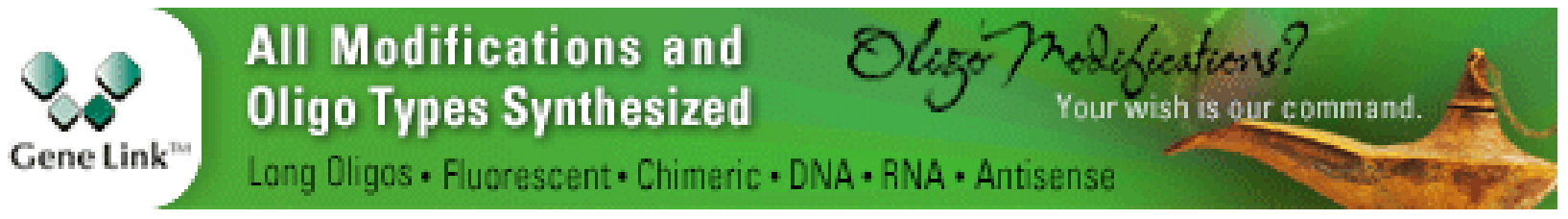

Copyright @ 2018 Cold Spring Harbor Laboratory Press; all rights reserved 